\title{
DIE ENTWICKLUNG DER REPUBLIK MALAWI ZWISCHEN FREIWILLIGER ABHÄNGIGKEIT UND AUTORITÅRER GRUNDBEDURFNISSTRATEGIE
}

\section{Musterland der Entwicklungshilfe oder internationaler Sozialhilfeempfänger?}

Der afrikanische Kleinstaat Malawi, halb so groß wie die Bundesrepublik Deutschland, gehört nach offizieller Einschätzung der Vereinten Nationen zur Gruppe der am wenigsten entwickelten Länder (LLDC). In den internationalen Statistiken wird dieses Land neben Staaten wie Benin, Niger, Ruanda oder Tschad als einer jener ,, hoffnungslosen Fälle“ geführt, die angesichts der scherenartig wachsenden Kluft zwischen Industrie- und Entwicklungsländern zu ,,Sozialhilfeempfängern“ der internationalen Staatengemeinschaft geworden sind.

In der Tat deuten die entwicklungsrelevanten Parameter des Landes nur auf ein geringes Wachstums- und Entwicklungspotential hin:

- Malawi ist ein ,,land-locked country“, das über keinen direkten Zugang zum Meer verfügt. Zur Abwicklung des Außenhandels ist das Land infolgedessen in hohem Maße auf das Wohlwollen seiner nachbarlichen Transitländer angewiesen.

- Die Topographie des Landes ist ungünstig: Mit seiner langgestreckten Ausdehnung entlang des Malawi-Sees ergeben sich Infrastruktur- und Kommunikationsprobleme zwischen den nördlichen und südlichen Landesteilen, die durch schwer überwindbare Gebirgsketten noch verstärkt werden. Bis Ende 1979 war die Seeschiffahrt das einzige Verkehrsmittel, mit dem der Güter- und Personenverkehr zwischen Nord und Süd in der Regenzeit aufrechterhalten werden konnte ${ }^{1}$.

- Im Gegensatz zu vielen anderen Entwicklungsländern verfügt Malawi nur über geringe Bodenschätze. Zu erwähnen sind lediglich Kohlevorkommen im äußersten Norden des Landes, die jedoch nicht von hoher Qualität sind; darüber hinaus gibt es verkehrsgünstig gelegene Vorräte von Bauxit, Graphit und anderen Erzen, deren Abbau sich in großem Umfang jedoch nicht lohnen würde. Kalkstein und Tonerde werden lediglich für den lokalen Bedarf der Baustoffproduktion abgebaut und verarbeitet.

- Aufgrund der geringen Bodenschätze ist das Industrialisierungspotential gering. Der Anteil des produzierenden Gewerbes - hauptsächlich Verarbeitung und Veredlung landwirtschaftlicher Produkte - liegt lediglich bei 11 Prozent des Bruttoinlandsproduktes ${ }^{2}$.

- Malawi ist ein Agrarland. Allerdings ist nur die Hälfte des malawischen Staatsgebietes landwirtschaftlich nutzbar. Mehr als 80 Prozent der Bevölkerung sind in der Landwirtschaft, zumeist noch in der Subsistenzproduktion, tätig. Mit einem Pro-Kopf-Einkommen von 140 \$ im Jahr gehört das Land statistisch zu der untersten Einkommensgruppe der afrikanischen Länder ${ }^{3}$.

\footnotetext{
1 Eine durch das Gebirge führende Allwetterstraße von Rumphi nach Chiweta ist mit bundesdeutscher Kapitalhilfe erst 1979 fertiggestellt worden. Vgl. Braun, Gerald - Weiland, Heribert, Soziale Aspekte von Staßenbaumaßnahmen. Versuch einer systematischen Erfassung am Beispiel eines Projektes im Norden Malawis, Freiburg, 1979 (unveröffentl. Forschungsbericht).

2 Vgl. Statistisches Bundesamt, Malawi, 1979, Länderkurzbericht, Wiesbaden, 1979, S. 7.

3 Ebenda.
} 
Diese ungünstigen Entwicklungsbedingungen sind jedoch nicht erst bekannt, seitdem die Entwicklungsökonomen der UN Armutsindikatoren definiert und Malawi der Kategorie der 4. Welt zugeordnet haben. Bereits während der Kolonialzeit wurde das damalige britische Protektorat Nyasaland als ,,imperial slum“ bezeichnet. Die britische Verwaltung war zu jener Zeit davon überzeugt, daß dieses Territorium, auf sich selbst gestellt, auf keinen Fall lebensfähig sein würde und somit nur als Arbeitskräftereservoir innerhalb einer Föderation mit Rhodesien (damals noch Süd- und Nordrhodesien) eine Zukunft besitze. Diese Einschätzung hat sich inzwischen fundamental gewandelt. Malawi wird heute als ,,Musterländle am Malawisee “4 oder als ,,prosperierender Polizeistaat ${ }^{\text {"5 }}$ gesehen, dem es seit seiner Unabhängigkeit im Gegensatz zu seinen Nachbarstaaten gelungen zu sein scheint, die Grundbedürfnisse der Masse seiner Bevölkerung zu befriedigen ${ }^{6}$.

Ob diese Einschätzung der sozio-ökonomischen Situation tatsächlich gerechtfertigt ist und welches die wichtigsten Determinanten der malawischen Entwicklung sind, wird im Rahmen konkurrierender Dependenzmodelle zu untersuchen sein.

\section{Zur strukturellen Abhängigkeit: Internationale Determinanten der malawischen} Wirtschaftsentwicklung

Da das interne Entwicklungspotential Malawis vergleichsweise gering ist, wird seine sozio-ökonomische Entwicklung entscheidend von der Zufuhr externer materieller und humaner Ressourcen bestimmt. Die daraus resultierende Abhängigkeit läßt sich im Rahmen konkurrierender Dependenzkonzepte analysieren, die idealtypisch nach Untersuchungsobjekt, Definition und Operationalisierung von Abhängigkeit klassifiziert werden können. Solche Konzepte sind etwa: externe Sensitivität; ungleicher Tausch; Fremdbestimmung; ungleiche Kosten- und Nutzenverteilung (vgl. Úbersicht 1).

Gemeinsam ist diesen Ansätzen, daß sie - im Unterschied zu den Interdependenztheorien Abhängigkeit als Komplement zu Herrschaft und Fremdbestimmung begreifen und im kausalen Zusammenhang mit Unterentwicklung konzeptualisieren ${ }^{7}$.

Eine Analyse von Abhängigkeit sieht sich im Falle Malawis mit zwei Schwierigkeiten konfrontiert: Zum einen ist das vorhandene empirisch-statistische Material unvollständig und inhomogen, zum anderen sind die vorliegenden Dependenzkonzepte (noch) nicht hinreichend operationalisiert, so daß nur vorläufige Aussagen über vermutete Zusammenhänge möglich sind.

\footnotetext{
4 Knopp, Guido, Magier in Malawi, FAZ vom 13. 4. 1978.

5 NZZ vom 9./10. 4. 1978.

6 ,,.. die malawische Landwirtschaft ernährt heute alle Landeskinder“, Schork, Erika, Der ,,gute Mensch“ mitder harten Hand, FR vom 4. August 1978 .

7 Vgl. Sieber, Margret M., Soziale Indikatoren für die Außenpolitik: Die Unabhängigkeit derSchweiz von ihrer Umwelt, Zürich, 1976, S. 47.
} 


\section{Ubersicht 1}

Entwicklungstheoretische Konzepte von Abhängigkei

Konzept

1. externe Sensitivität ${ }^{9}$
Definition und Operationalisierung

Abhängigkeit als externe Sensitivität

bestimmt durch:

- Ausmaß des Außenhandelsanteils am BSP

- Ausmaß des Export-/Importanteils am BSP

- Ausmaß der Partnerkonzentration im Ex- und Import

- Ausmaß der Partnerkonzentration im Kapitalverkehr

- Ausmaß der $\mathrm{Zu}$ - und Abwanderung von Arbeitskräften

2. ungleicher ${ }^{10}$

Abhängigkeit als Austausch ungleicher Güter

Austausch bestimmt durch:

- Ausmaß der Ungleichheit ausgetauschter Güter hinsichtlich Preis und Qualität

- Ausmaß der Produktionskonzentration im Ex- und Import

- Entwicklung des Leistungsbilanzsaldos

- Entwicklung von Auslandsverschuldung und Schuldendienst

8 Vgl. hierzu: Sieber, Margret, M. Soziale Indikatoren für die Außenpolitik. Die Abhängigkeit der Schweiz von ihrer Umwelt, Zürich 1976, S. 48 
Konzept

3. Fremdbestimmung ${ }^{11}$

Definition und Operationalisierung

\begin{abstract}
Abhängigkeit als Verlust an Autonomie bestimmt durch:

- Anteil ausländischen Kapitals an gesamtwirtschaftlicher Investitionsquote

- Ausmaß der Penetration, d. h. Ausmaß der Teilnahme von Ausländern am Entscheidungsprozeß

- Ausmaß der fremden Kontrolle über Entscheidungen

- Ausmaß der Einschränkung von Entscheidungsoptionen und Verhaltensalternativen
\end{abstract}

4. ungleiche Kosten ${ }^{12}$ Abhängigkeit als ,Ausbeutung',
als ungleiche Kosten-Nutzen-Verteilung
bestimmt durch: (,Ausbeutung')

- Ausmaß der Ungleichheit der Inter-Akteur-Effekte, d. h. Ausmaß der Vergrößerung der internationalen Positionsunterschiede

- Ausmaß der Ungleicheit der Intra-Akteur-Effekte, d. h. Ausmaß der Vergrößerung der nationalen Positionsunterschiede 
Versucht man unter diesen Einschränkungen das vorhandene Material den verschiedenen Ansätzen von Abhängigkeit zuzuordnen, so kommt man zu folgenden Ergebnissen (vgl. auch Ubersicht 2):

\subsection{Externe Sensitivität}

Im internationalen Vergleich besonders bemerkenswert ist der extrem hohe und weiter ansteigende Anteil des malawischen Außenhandels am Bruttosozialprodukt (BSP). Er kann als Korrelat der geringen Größe des Landes betrachtet werden ${ }^{13}$. Entsprechend ist die wirtschaftliche Entwicklung Malawis zunehmend an den Wachstums- und Konjunkturverlauf seiner Haupthandelspartner, wenngleich zeitlich versetzt, gekoppelt.

Unter ihnen nehmen mit der ehemaligen Kolonialmacht Großbritannien auf der Exportsowie der Republik Südafrika auf der Importseite kapitalistisch organisierte Staaten eine dominierende Stellung ein. Der Außenhandel mit sozialistischen Industrieländern sowie mit Staaten der 3. Welt ist - Sambia ausgenommen - ohne nennenswerte Bedeutung.

Seit 1964 hat Malawi beim Export seine Partnerstruktur geringfügig diversifiziert, beim Import hingegen in extremem MaßeaufSüdafrika konzentriert (Zunahme von 5,6 auf 42,9 Prozent), das die Position Rhodesiens in der früheren kolonialen Föderation eingenommen hat. Noch höher war nach der Unabhängigkeit die Partnerkonzentration im Kapitalverkehr, da mehr als die Hälfte aller Zuflüsse an öffentlichem und privatem Auslandskapital auf Großbritannien entfielen. Es ist Malawi gelungen, diese einseitige Abhängigkeit beim Kapitalimport durch Erschließung insbesondere multilateraler Vergabestellen abzubauen, deren Anteil inzwischen bei knapp einem Drittel liegt.

Ähnlich erfolgreich wie die schrittweise Loslösung von britischem Kapital war der Versuch, die traditionelle Wanderarbeit nach Südafrika, Rhodesien (Zimbabwe), Sambia und Moçambique zu verringern. Die Reintegration von - je nach Quelle ${ }^{\mathbf{1 4}}-200000$ bis 300000 Wanderarbeitern in die heimische Volkswirtschaft gelang im Zeichen einer guten Binnenkonjunktur ohne nennenswerte Vergrößerung der Arbeitslosigkeit. Im Juli 1977 hat die malawische Regierung das Anwerbeverbot von 1974 jedoch wiederaufgehoben ${ }^{15}$.

13 Kuznets hat für Kleinstaaten sogar die Entwicklung einer eigenen Wirtschaftstheorie vorgeschlagen. Vgl. Kuznets, Simon, Six Lectures on Economic Growth, Illinois, 1959, p. 90. Khalaf hingegen kommt in einer multiplen Korrelationsanalyse zu dem Ergebnis, daß ,,neither the dependence on trade of small countries nor their commodity and geographic export concentration are necessarily important factors in economic grwoth and economic development". Khalaf, Nadim, G., Country Size and Economic Growth and Development, in: The Journal of Development Studies, Vol. 16, No. 1, October 1979, p. 67.

14 Weyl beziffert während der Jahre 1968-71 die Zahl der Wanderarbeiter allein nach Südafrika mit 358 612, was - bei gegenwärtig 20000 - über 300000 ,,Rückkehrer" bedeuten würde. Vgl. Weyl, Ulrich, Wanderarbeit und Unterentwicklung, in: Wilson, Francis, u. a., Wanderarbeit im Südlichen Afrika, Bonn, 1976, S. 202. In einer Feasibility-Studie wird von ,,some 180000 migrant workers reported returning to the country between 1974 and 1976" gesprochen. Republic of Malawi, Jenda Mzuzu Road, Feasibility Study Draft. Final Report, Main Text, March 1979, p. 27.

15 Vgl. Bundesstelle für Außenhandelsinformation, Malawi. Wirtschaftliche Entwicklung 1977, Köln, 1978, S. 2. Legum stellt dazu fest: "Malawian labour virtually dried up; only 207 Mala wians were on the mines in April 1977, though the number rose quite significantly again by the end of the year." Legum, Colin (ed.), Africa Contemporary Record, 1977-78, New York and London, 1979, B 927. 
Übersicht 2

Indikatoren von Abhängigkeit der Volkswirtschaft der Republi

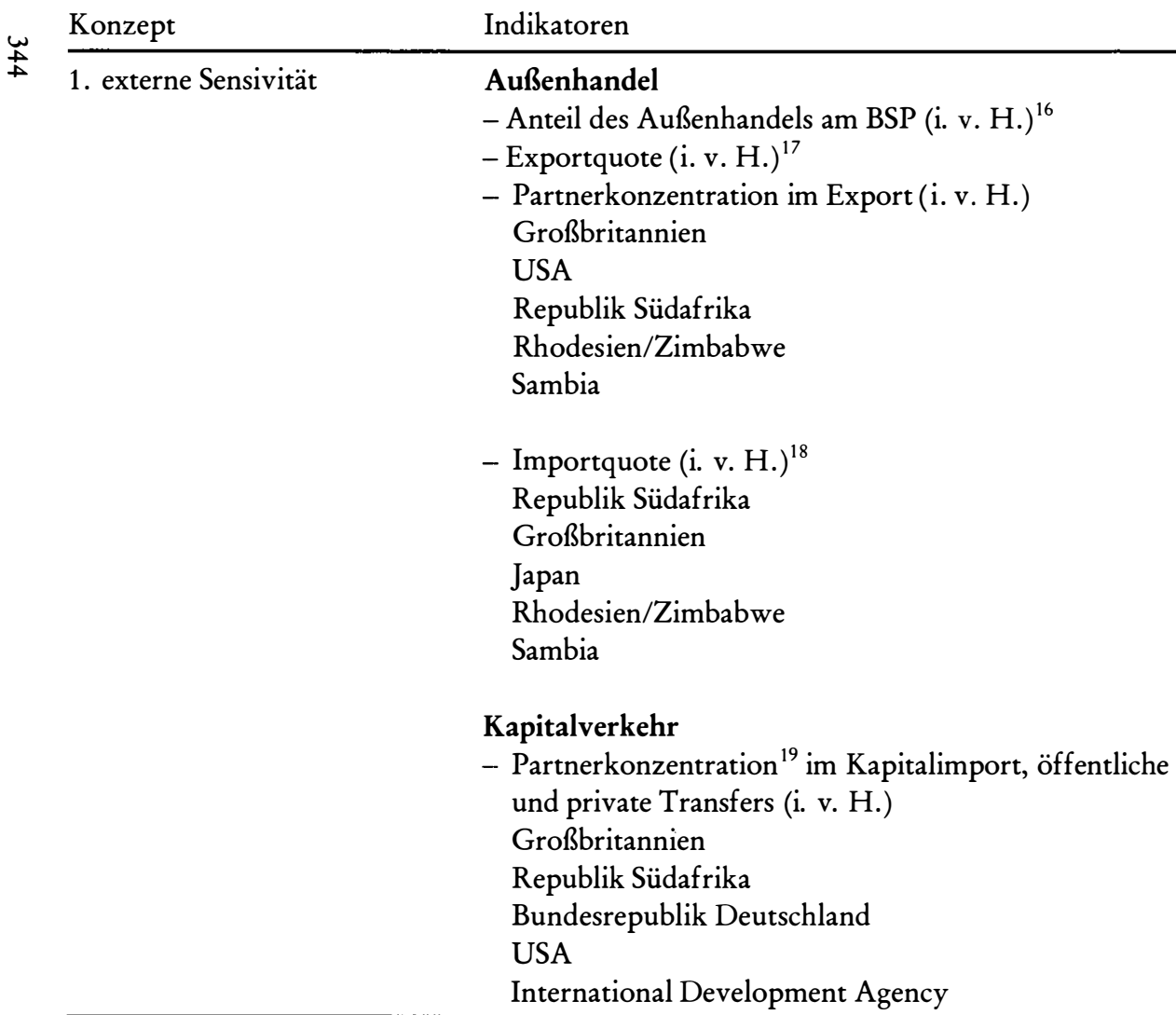

\footnotetext{
16 Alle Außenhandelswerte berechnet nach: Malawi Statistical Yearbook 1976, Zomba 1977, p. 178 f.; Malawi Monthly Statistical Bulletin, April 1979, Länderkurzbericht Malawi 1979, Wiesbaden 1979, S. 17

17 Anteil der Exporte a m Bruttosozialprodukt in v. H. einschließlich Re-Exporte, f. o. b. Werte

18 Anteil der Importe am Bruttosozialprodukt in v. H., c. i. f. Werte

19 Anteil an privaten und öf entlichen Auslandsschulden in v. $\mathrm{H}$.

20 Fischer, Knut, M. und Weyl, Ulrich Art. Malawi . . a. a. O., S. 335 (für 1964-1970)

21 Für 1973, Bundesstelle für Außenhandelsinformation, Malawi, Wirtschaftliche Entwicklung 1977, Köln 1978, S. 42

22 Ebenda 
Fortsetzung Übersicht 2

Indikatoren von Abhängigkeit der Volkswirtschaft der Republi

Indikatoren

\section{Migration}

- Malawische Wanderarbeiter (i. 1000)

- Anteil an männl. Erwerbstätigen (i. v. H. ${ }^{26}$

- Anteil an Lohn- und Gehaltsempfängern ${ }^{11}$

in festem Arbeitsverhältnis (i.v. H.)

2. ungleicher Austausch

Exportstruktur (i. v. H. $)^{27}$

- Agrarprodukte darunter:

Tabak

Tee

Erdnüsse

Zucker

- mineralische Roh- und Brennstoffe

- Fertigwaren

Importstruktur (i. v. H. $)^{28}$

- Agrarprodukte

- mineralische Roh- und Brennstoffe

- Fertigwaren darunter:

Maschinenbau- und elektrotechnische Erzeugnisse, Fahrzeuge

23 Für 1966. Weyl, Ulrich Wanderarbeit im südlichen Afrika ... a. a. O., S. 202

24 Für 1968-1971, nur in die Republik Südafrika, für Rh.odesien/Zimbabwe, Mocambique und Sambia keine Angaben, vgl. Weyl, Ulrich Wanderarbeit

25 Anfang 1978. Bundesstelle für Außenhandelsinformation, Malawi . . . a. a. O., S. 2. Vgl. dagegen: „Die Zahl der im Ausland lebenden Malawier wi Goldminen arbeiten“. Statistisches Bundesamt, Statistik des Auslandes. Länderkurzbericht Malawi 1979, ... a. a. O., S. $5 \mathrm{f}$

26 Berechnet nach: Statistisches Bundesamt ... a. a. O., S. 14

27 Anteil der Produktgruppen und Produkte am Gesamtexport ohne Re-Export in v. H., f. o. b. Werte. Angaben 1964 und 1970 berechnet nach: Ma nach: Statistisches Bundesamt . . . a. a. O., S. $17 \mathrm{f}$

28 Anteil der Produktgruppen und Produkte am Gesamtimport in v. H., c. i. f. Werte.

29 Für 1968, f. o. b. Wert, berechnet nach: Malawi Statistical Yearbook 1976 . . a. a. O., p. 182

30 Berechnet nach: Malawi Statistical Yearbook 1976 . . . a. a. O., p. 182

31 Für 1976. Weltbank (Hirsg.) Weltentonickingsbericht 1979, 339 a. a. O. . . 662 
Fortsetzung Ubersicht 2

Indikatoren von Abhängigkeit der Volkswirtschaft der Republik

\begin{tabular}{ll} 
\pm & Indikatoren \\
\hline & terms of trade (1970=100 \\
& Leistungsbilanzsaldo (i. Mill. Dollar) \\
& öffentliche Auslandsverschuldung ${ }^{37}$ \\
& - in Mill. Dollar \\
& - in v. H. des Bruttosozialprodukts
\end{tabular}

Schuldendienst in v. H. der Waren- und Dienstleistungsexporte ${ }^{38}$ Bruttowährungsreserven (in Mill. Dollar) ${ }^{39}$

3. Verlust an Autonomie (,Fremdbestimmung')

\section{Anteil ausländischen Kapitals}

Entwicklungsprogramm (i. v. H.) darunter:

International Development Agency

Großbritannien

USA

Anteil ausländischer ${ }^{42}$ Arbeitskräfte an Erwerbstätigen in dauerhaftem Arbeitsverhältnis (i. v. H.)

Anteil ausländischer Arbeitskräfte an Erwerbstätigen in entscheidungsrelevanten Positionen (i. v. H.)

32 Für 1967. Malawi Statistical Yearbook 1976 ... a. a. O., p. 186

33 Bundesstelle für Außenhandelsinformation. Weltwirtschaft am Jahreswechsel, Malawi 1979 . . 2. 2. O., S. 4

34 Für 1965. Berechnet nach: Malawi Statistical Yearbook 1976 . . . a. a. O., p. 187

35 Berechnet nach: Malawi Statistical Yearbook 1976 ... a. a. O., p. 187

36 Berechnet nach: Statistisches Bundesamt ... a. a. O., S. 24

37 Weltbank (Hrsg.) Weltentwicklungsbericht 1979 ... 2. 2. O., S. 172

38 Ebenda, S. 168

39 Ebenda, S. 168

40 Für Dreijahresplan 1975/76 bis 1977/78. Statistisches Bundesamt . . a. a. O., S. 24

41 Für Dreijahresplan 1979/80 bis 1981/82. Statistisches Bundesamt . . . 2. 2. O., S. 24

42 Geschätzt nach: Bundesstelle für Außenhandelsinformation, Mala wi. Wirtschaftt. Entwicklung 1977 . . a. a. O., S. 42

43 Berechnet nach: Statistisches Bundesamt ... a. 2. O., S. 5 und S. 14

44 Fivene Srhärininu 
Fortsetzung Ubersicht 2

Indikatoren von Abhängigkeit der Volkswirtschaft der Repub

\begin{tabular}{ll} 
Konzept & Indikatoren \\
\hline $\begin{array}{l}\text { 4. ungleiche Kosten- } \\
\text { Nutzen-Verteilung }\end{array}$ & Sektorale Einkommensverteilung ${ }^{45}$ \\
(,Ausbeutung') & bei Lohn- und Gehaltsempfängern \\
& (Bruttomonatsverdienste in Malawie Kwacha) \\
& - Landwirtschaft \\
& - Bergbau \\
& - Verarbeitendes Gewerbe darunter: \\
& - Nahrungsmittelindustrie \\
& - Fahrzeugbau \\
& - Baugewerbe \\
& - Verkehr und Nachrichtenübermittlung \\
& Personelle Einkommensverteilung ${ }^{46}$ \\
& bei Lohn- und Gehaltsempfängern \\
& - untere Einkommenskategorie (unter 599 MK pro Jahr) \\
& in v. H. aller Lohn- und Gehaltsempfänger \\
& - mittlere Einkommenskategorie (600-1499 MK) \\
& in v. H. aller Lohn- und Gehaltsempfänger \\
& - obere Einkommenskategorie (über 1500 MK) \\
& in v. H. aller Lohn- und Gehaltsempfänger
\end{tabular}

w 45 Statistisches Bundesamt... a. a. O., S. 22 (für 1973 und 1977)

$\underset{V}{+} 46$ Zahl der Personen in jeweiligen Einkommenskategorien, berechnet nach: Malawi Statistical Yearbook 1976, . . a. a. O., p. 71 (für 1971 und 1975) 


\subsection{Ungleicher Austausch}

Die Außenhandelsstruktur Malawis ist typisch für das dominierende Spezialisierungsmuster im System der internationalen Arbeitsteilung. Knapp 80 Prozent (1977) der Gesamtexporte entfallen auf die subtropischen Agrarprodukte Tabak und Tee, während ca. 75 Prozent (1977) der Importe aus industriellen Fertigwaren bestehen (vgl. Ubersicht 2). Im Unterschied zu anderen agrarischen Entwicklungsländern hat Malawi seine Exportstruktur nicht diversifiziert, sondern zunehmend auf die „Duokulturen“ Tabak und Tee konzentriert. Diese chancen- wie risikoreiche Exportspezialisierung profitierte in den siebziger Jahren vom weitgehenden Ausfall Rhodesien/Zimbabwes als Tabakanbieter auf den Weltmärkten sowie von steigenden Weltmarktpreisen für Tee. Infolgedessen verbesserten sich die (barter) terms of trade, die im 2. Quartal 1975 aufgrund inflationierender Erdöl- und Fertigwarenimporte auf das Rekordtief von $67,25^{47}$ (Basis $1970=100$ ) gesunken waren, auf $94,06^{48}$ in 1977. Allerdings scheint es sich hierbei um eine historisch einmalige Konstellation gehandelt zu haben. Der absehbare Konkurrenzdruck auf den Weltmärkten für Tabak (Zimbabwe) und Tee (Indien, Sri Lanka) einerseits und steigende Importpreise bei Erdöl und Erdölderivaten andererseits dürften mittelfristig die Handelsbilanz wieder stark passivieren. Bereits 1978 verschlechterten sich die terms of trade um etwa 10 Punkte auf $84,7^{49}$. Entsprechend stieg das Leistungsbilanzdefizit geradezu dramatisch von 34 Mio. Dollar(1977) auf 156 Mio. Dollar (1978) ${ }^{50}$. Da diese Entwicklung in der ersten Jahreshälfte 1979 anhielt, erließ die malawische Regierung im August 1979 umfassende Importbeschränkungen.

Jedoch hatte Malawi - wie auch die steigenden Bruttowährungsreserven ausweisen - in der Vergangenheit keine Schwierigkeiten, im Ausland öffentliche und private Kapitalgeber zu finden, welche die chronisch defizitäre Leistungsbilanz des Landes mehr als ausglichen ${ }^{\mathbf{5 1}}$. „,Die seit anderthalb Jahrzehnten anhaltende innenpolitische Stabilität . . . sowie die großzügigen Finanzierungskonditionen für ausländische Investitionen lassen den Zustrom von Uberseekapital nach Malawi nicht abreißen. "52

Die bi- und multilaterale Entwicklungshilfe wird in der Regel zu günstigen Konditionen vergeben $^{53}$, so daß der Schuldendienst in Relation zum Exporteinkommen sogar abgenommen hat (vgl. Ubersicht 2). Hinzu kommt, daß wichtige Gläubigerländer wie Großbritannien und die Bundesrepublik einen bilateralen Schuldenerlaß vereinbarten ${ }^{54}$. Dennoch zeichnen sich gegenwärtig bereits Liquiditätsengpässe ab, da Malawi seit 1977/78 kurzfristige Kredite zu kommerziellen Bedingungen bei amerikanischen Geschäftsbanken aufnehmen mußte ${ }^{55}$. Mit Fälligwerden dieser Kredite wird der Schuldendienst für Auslandsschulden erheblich ansteigen. Er dürfte, falls keine Anschlußfinanzierung gefunden werden sollte, in Malawi die verstärkte Suche nach neuen Exportmärkten und -produkten auslösen und eine weitere Verschärfung der Importrestriktionen notwendig machen ${ }^{\mathbf{5 6}}$.

47 Malawi Statistical Yearbook, 1976, Zomba, 1977, p. 186.

48 Mitteilungen der Bundesstelle für Außenhandelsinformation, Weltwirtschaft a m Jahreswechsel, Malawi, 30. Jg., Februar 1980, S. 4.

49 Ebenda. Dies nicht zuletzt deswegen, weil sinkende Kaffeepreise einen kontinuierlichen Preisverfall bei Tee auslösten.

50 Ebenda.

51 So auch Ashford: "So efficient and diligent has Malawi proved itself to be in developing its own resources that aid bodies such as the World Bank, Commonwealth Development Corporation and European development funds almost fall over each other in the rush to give Malawi loans and grants." Ashford, Nicholas, The adoration . . . a. a. O.

52 Blick durch die Wirtschaft, FAZ vom 5. Oktober 1979.

53 So gewährte die International Development Agency (IDA) Anfang 1979 Malawi ein zinsloses Darlehen über eine Laufzeit von 50 Jahren mit 10 tilgungsfreien Jahren, vgl. Standard Chartered Review, February 1979, p. 9.

54 Großbritannien in Höhevon 52 Mio. MK und die Bundesrepublik in Höhevon 43 Mio. MK, vgl. Standard Chartered Review, July 1979. 1 US-Dollar = 0,12 Malawi Kwacha (MK).

5525 Mio. Dollar bei der Chase Manhatten Bank und 10 Mio. Dollar bei der US-Citybank, vgl. Mitteilungen der Bundesstelle für Außenhandelsinformation, Weltwirtschaft am Jahreswechsel ..., a. a. O., S. 2.

56 Vgl. auch Republic of Malawi, Jenda-Mzuzu Road..., a. a. O., p. 34, und Malawi: Reverberations, in: Africa Confidential, Vol. 21, No. 7, 1980, p. 5. 


\subsection{Abhängigkeit als Verlust an Autonomie (,,Fremdbestimmung")}

Die externe Sensitivität Malawis bei strukturell ungleichen Austauschverhältnissen läßt vermuten, daß das Land bei der Finanzierung und Durchführung seiner Entwicklungsprogramme in starkem Maße vom Ausland abhängig ist.

Die staatlichen Investitionen, die knapp zwei Drittel der gesamtwirtschaftlichen Investitionsquote ausmachen, werden zu 87 Prozent mit ausländischen Mitteln finanziert ${ }^{57}$. Die wirtschaftliche Entwicklung Malawis ist daher - neben den Devisenerlösen aus Exporten vor allem nach Großbritannien und den USA - wesentlich von öffentlichen Entwicklungshilfeleistungen, ebenfalls Großbritanniens und der USA, abhängig (vgl. Úbersicht 2).

Zwar war Malawi erfolgreich bemüht, seine finanzielle Abhängigkeit von Großbritannien durch Diversifizierung seiner Gläubigerstruktur ${ }^{58}$ abzubauen, jedoch dürfte die Republik Südafrika einen erheblichen, in den Statistiken nicht enthaltenen Anteil an den öffentlichen Gesamtleistungen haben. Bekannt ịst, daß Südafrika langfristige Kredite zum Aufbau der neuen malawischen Hauptstadt Lilongwe (8 Mio. Rand), zur Errichtung von Getreidesilos (11 Mio. Rand) und zum Ausbau des Eisenbahnnetzes (10 Mio. Rand) gewährt hat ${ }^{59}$.

Noch höher ist der Beitrag südafrikanischer Firmengruppen zur privatwirtschaftlichen Investitionstätigkeit im Lande einzuschätzen. Neben britischen Unternehmen wie der Imperial- und der Lonrho-Gruppe sind südafrikanische Firmen im Straßenbau, in der Landwirtschaft, der Süßwasserfischerei und im medizinischen Sektor ,,stark engagiert “60. Der relativ kleine Binnenmarkt und die geringen Absatzchancen in den Nachbarstaaten lassen jedoch größere kommerzielle Investitionsvorhaben lediglich bei den landwirtschaftlichen Weltmarktprodukten rentabel erscheinen.

Was die personellen Abhängigkeiten des Landes anlangt, so stellen die ca. 6000 erwerbstätigen Ausländer ${ }^{61}$ nur rund 2 Prozent der Erwerbstätigen in ständigem Arbeitsverhältnis, dürften aber überwiegend in mittleren und oberen Managementpositionen tätig sein. Es scheint daher nicht unrealistisch, davon auszugehen, daß je nach Tätigkeitsbereich 25 bis 50 Prozent aller wichtigen Entscheidungspositionen des Landes von Ausländern besetzt sind. Im politisch-administrativen Sektor hat sich dabei eine, für zahlreiche Entwicklungsländer typische Variante des Stab-Linien-Systems herausgebildet. Während die - formal entscheidungsberechtigte - Linie von der heimischen Elite besetzt ist, rekrutieren sich die - materiell entscheidenden - Stäbe überwiegend aus ausländischen Experten.

Im privaten Sektor ist vor allem auf den Einfluß ausländischer Plantagenbesitzer und Betriebsleiter in der Landwirtschaft aufmerksam zu machen. In jüngerer Zeit haben hohe malawische Politiker und Beamte als, absentee landlords' größere Plantagen (insbesondere für Tabak, Tee und Zuckerrohr) eröffnet bzw. übernommen ${ }^{62}$, die unter anderem von Briten und weißen Rhodesiern ${ }^{63}$ geleitet werden.

\footnotetext{
57 Dies gilt für den laufenden Dreijahresplan 1979-1982. Vgl. Statistisches Bundesamt. Statistik des Auslandes. Länderkurzbericht, Malawi, 1979, Wiesbaden, 1979, S. 24.

58 Zu den neuen Finanzierungsquellen zählen: die International Development Agency, verschiedene Sonderfonds der UN, Sonderziehungsrechte des IMF, die Commonwealth Development Corporation, der Europäische Entwicklungsfonds, der OPEC-SpecialFund und die Afrikanische Entwicklungsbank.

59 Vgl. Blick durch die Wirtschaft vom 5. Oktober 1979.

60 Ebenda.

61 Es gibt in Malawi etwa 20000 nicht-afrikanische Ausländer, davon 12000 Inder und Pakistani. Vgl. Statistisches Bundesamt . . ., a. a. O., S. 5. Andere Quellen sprechen von 10000 nicht-afrikanischen Ausländern, davon 6000 Europäer und $4000 \mathrm{Asiaten}$. Vgl. Bundesstelle für Außenhandelsinformation, Malawi, 1977 . ., a. a. O., S. 1.

62 Vgl. Fischer, Knut M., und Weyl, Ulrich, Malawi, in: Nohlen, Dieter, und Nuscheler, Franz (Hrsg.), Handbuch der Dritten Welt, Bd. 2, Halbband I, Hamburg, 1976, S. 334.

63 Die Abwanderung der Weißen aus Rhodesien/Zimbabwe in den letzten Jahren betrug: 7100 (1976), 10000 (1977), 13 700 (1978), 8300 (Jan.-Sept. 1979), NZZ vom 17. Januar 1980.
} 


\subsection{Abhängigkeit als ungleiche Kosten-Nutzen-Verteilung (,,Ausbeutung“)}

Die skizzierten Abhängigkeiten dürften, da sie gleichsam kumulativ wirken, inter- wie intragesellschaftliche Prozesse ungleicher Kosten-Nutzen-Verteilung induzieren, die in der Literatur auch als Ausbeutungsprozesse bzw. als Prozesse struktureller Bereicherung und Verarmung bezeichnet werden.

Von der definitorischen Unschärfe des Begriffs ,,Ausbeutung“ abgesehen, lassen sich derartige Mechanismen in Ermangelung geeigneten empirischen Materials für Malawi nur näherungsweise bestimmen.

International führen sinkende terms of trade ceteris paribus zu einem Ressourcentransfer, der noch durch Zinszahlungen, Gewinntransfers und Fluchtgelder verstärkt werden kann. Das günstige Investitionsklima ${ }^{64}$ im Lande hat jedoch bislang zu Kapitalimporten, zum Teil in Form von Schenkungen, geführt, welche die defizitäre Leistungsbilanz des Landes mehr als ausgleichen. Dank günstiger Konditionen der Kapitalimporte hat der Schuldendienst, und damit ein wesentlicher Teil des Kapitalexports Malawis, relativ gesehen, sogar abgenommen.

Die strukturelle Abhängigkeit Malawis hat also - paradoxerweise - trotz adverser terms of trades und chronisch defizitärer Leistungsbilanz intergesellschaftlich eher zu einer Bereicherung als zu einer Verarmung des Landes geführt.

Die intragesellschaftlichen Effekte dieses Prozesses lassen sich wegen der unzureichenden Einkommens- und Vermögensstatistik nicht exakt nachweisen. Immerhin fällt bei den abhängigen Erwerbstätigen mit festem Arbeitsverhältnis die große und weiter wachsende Ungleichheit der monatlichen Durchschnittseinkommen in den verschiedenen Wirtschaftssektoren auf ${ }^{65}$. Offenbar wird die Konkurrenzfähigkeit auf den Weltmärkten durch entsprechend niedrige Löhne im Agrarsektor gesichert. Hinweise auf die ungleiche Einkommensund Vermögensverteilung in der Gruppe der selbständig Erwerbstätigen erlaubt eine Analyse der Einkommens- und Besitzverhältnisse im dominierenden Landwirtschaftssektor. Da der staatliche Agricultural Development and Marketing Board (ADMARC) gegenüber den Kleinbauern (small-holder) mit cash-crop-Produktion über ein Liefermonopol bei Düngemitteln und Saatgut sowie über ein Ankaufsmonopol bei Agrarprodukten verfügt, bestimmt seine Preispolitik wesentlich die Einkommens- und Vermögensbildung der Kleinbauern außerhalb des Subsistenzsektors. Die Kritik der Bauern ${ }^{66}$ an den niedrigen Ankaufspreisen und hohen Gewinnen von ADMARC deutet darauf hin, daß zu ihren Lasten ein Zwangssparprozeß stattfindet, von dem möglicherweise auch die, dem Staatspräsidenten nahestehende, Board-Verwaltung profitiert.

Die - im Besitz britischer oder mala wischer Privatpersonen befindlichen - 120 Großbetriebe (estates) in der Tee- und Tabakproduktion umfassen mit ca. 2,5 Prozent ${ }^{67}$ nur einen geringen Teil der landwirtschaftlichen Nutzfläche, verfügen aber über einen hohen Maschinenbesatz, Lohnarbeit und zum Teil ausländisches Management aus weißen Rhodesiern. 60 Prozent aller Düngemittel ${ }^{68}$ wird von diesen 120 Betrieben verbraucht, die - bei entsprechend hohen

64 Hierzu zählen auch die Garantie der ,,Repatriierung “ von Gewinnen, die Konvertibilität der Währung und der Verzicht auf Nationalisierung ausländischer Privatinvestitionen.

65 Allgemeine Hinweise hierzu geben Fischer, Knut M., und Weyl, Ulrich, Malawi . ., a. a. O., S. $332 \mathrm{ff}$.

66 "Some farmers complain that the organization makes a handsome profitfrom its operations and could afford to pay more", The Times, 27. September 1978.

67 Fischer, Knut M., und Weyl, Ulrich, Malawi ..., a. a. O., S. 334

68 Während 1970/71 an Smallholder-Betriebe noch 22845 short tons Düngemittel und an Estates 18726 short tons verkauft wurden, beliefen sich die Verkäufe 1976/77 bereits auf 33664 bzw. 47194 short tons. Bundesstellefür Außenhandelsinformation, Malawi, 1977, a. a. O., S. 21 
Flächenproduktivitäten - über 60 Prozent (1977) aller Exporte Malawis auf sich vereinigen $^{69}$.

Von Staatspräsident H. Banda und hohen Parteifunktionären ist bekannt, daß sie einige der größten Plantagen in der Zentralregion des Landes persönlich besitzen ${ }^{\mathbf{7 0}}$.

Darüber hinaus ist die Regierung ,, über den staatlichen Konzern Press Holdings Ltd. in vielen Bereichen der landwirtschaftlichen und industriellen Produktion beteiligt. Mit der Press Holdings-Tochter General Farming Company werden 30 Prozent der landwirtschaftlichen Aktivitäten kontrolliert. Der größte Teil der Aktien der Press Holdings Ltd. befindet sich im Privatbesitz von Hastings Banda"71. Es besteht eine fast totale Verflechtung aller Wirtschaftszweige mit dem Präsidenten und der Partei, der Malawi Congress Party.

Zusammenfassend ist festzuhalten, daß die strukturelle Abhängigkeit des Landes von den internationalen Waren- und Kapitalmärkten seit der politischen Unabhängigkeit laufend gewachsen ist. Hierzu hat die risikoreiche Spezialisierung auf die zwei Exportprodukte Tabak und Tee beigetragen, eine Exportstrategie, die sich primär aus den internen Macht- und Eigentumsverhältnissen Malawis erklären läßt. Andererseits ist es der Republik Malawi gelungen, ihre einseitige finanzielle und personelle Abhängigkeit von der ehemaligen Kolonialmacht Großbritannien zu verringern und den Arbeitskräfteexport früherer Jahre durch Kapitalimporte zu substituieren.

3. Aufgezwungene oder freiwillige Abhängigkeit:

Die Persönlichkeit des Präsidenten als zentrale Determinante des politischen Prozesses

Im Falle Malawis lassen sich, sofern das vorliegende empirische Material eine derartige Aussage erlaubt, alle vier Formen der Abhängigkeit eindeutig nachweisen. Weniger eindeutig nachweisen lassen sich anhand der skizzierten Konzepte jedoch die Determinanten, die zu dieser Abhängigkeit führten. Das methodologische Instrumentarium ist nicht weitreichend genug, um die auslösenden Ursachen der Dependenz zu erklären. Die vorliegenden Konzepte neigen vielmehr zu einer mechanistisch-ökonomistischen Interpretation und verstellen damit die Sicht für psychosoziale, kulturelle und politische Einflußfaktoren.

Die Tatsache, daß trotz der Rohstoffarmut ein internationaler Ressourcentransfer zugunsten Malawis stattgefunden hat, legt die Vermutung nahe, daß es sich im Falle dieses Landes nicht um eine von außen aufgezwungene Abhängigkeit, sondern eher um eine von der politischen Führung Malawis selbst gewünschte und bewußt kalkulierte Dependenz handelt ${ }^{72}$. Im Gegensatz zur typischen Abhängigkeit rohstoffreicher Entwicklungsländer, die zum Teil verzweifelt gegen die überstarke Marktmacht der Industrieländer anzukämpfen versuchen, scheinen die Machthaber in Malawi durchaus bemüht zu sein, abhängig zu werden und abhängig zu bleiben - auch wenn das wirtschaftliche Interesse der großen Industriestaaten an diesem Land vergleichsweise gering ist. Es ist vor allem der Staatspräsident Dr. Hastings Kamuzu Banda, der die Anlehnung an die westlichen Industriestaaten immer wieder propagiert und kraft seines Amtes und seiner Machtfülle auch durchgesetzt hat. Sein dominierender Einfluß auf die neueste Geschichte des Landes läßt sich exemplarisch an seiner Rolle in

69 Ebenda, S. 21.

$70 \mathrm{Vgl}$. Ashford, Nicholas, The adoration . .., a.a. O., und Frank, Michael, Alleinherrscher auf Lebenszeit, in: SZ, 14./15. 8. 1980. 71 Blick durch die Wirtschaft, FAZ vom 5. Oktober 1979.

$72 \mathrm{Vgl}$. dazu den am Beispiel Ruandas nachgewiesenen Erklärungsversuch freiwilliger Abhängigkeit von Hanf, Theodor, Astochastische Dependenz als Entwicklungshindernis, in: Schmölz, Franz-Martin (Hrsg.), Christlicher Friedensbegriff und europäische Friedensordnung, München/Mainz, 1977, S. 151-164. 
der Entkolonisierungsphase und bei der Ausrichtung eines politischen Kurses nach der Unabhängigkeit Malawis aufzeigen.

Eine eigenständige Unabhängigkeitsbewegung entstand in Nyasaland erst ziemlich spät. Der Nyasaland African Congress (NAC) als politische Artikulationsform schwarzen Willens nach Unabhängigkeit und Selbstbestimmung wurde erst in den 40er Jahren gegründet. Den Vertretern in dieser Partei gelang es bis in die späten 50er Jahre nicht, eine Unterstützung breiter Bevölkerungskreise zu erreichen. Dabei erwies sich vor allem die Jugend und Unerfahrenheit der ersten Politikergeneration, die der souveränen Routine der Kolonialadministration nur wenig entgegenzusetzen hatte, als ein großes Handikap ${ }^{73}$. Insofern fiel Banda, der als einer der ganz wenigen Schwarzen Nyasalands eine Universitätsausbildung absolviert hatte, eine wichtige Funktion zu. Trotz seines fast 40jährigen Auslandsaufenthaltes in Südafrika, den USA, Großbritannien und Ghana hatte er mit einflußreichen Persönlichkeiten in Nyasaland Kontakt gehalten und immer wieder in der Offentlichkeit zur politischen Entwicklung seines Heimatlandes Stellung genommen. Die von der Kolonialverwaltung betriebene ökonomische und politische Föderation der drei Territorien Südrhodesien, Nordrhodesien und Nyasaland hatte er besonders kritisiert. "The proposed federation will not be a commonwealth in which Europeans und Africans will be equal partners, but a slave state in which the Europeans will be the privileged masters and the Africans the privileged serfs."74 Sein Rat und sein Urteil wurden nicht nur in Nyasaland, sondern auch in London, wenn auch widerwillig, gehört und beachtet. So ist es nicht verwunderlich, daß Banda trotz seines langjährigen Exils in die Rolle einer legendären grauen Eminenz des Unabhängigkeitskampfes hineinwuchs, dessen Rückendeckung und Unterstützung für die Parteispitze vor Ort geradezu unentbehrlich wurde.

1958, als die Auseinandersetzungen um das Fortbestehen der Föderation immer heftiger wurden, wurde er von Repräsentanten des NAC nach Hause zurückgerufen, um an der Spitze der Unabhängigkeitsbewegung aktiv am Dekolonisierungsprozeß teilzunehmen. Allerdings erwartete kaum einer der aktiven Politiker jener Zeit, daß der damals 60jährige noch eine lange politische Karriere vor sich hätte. Man glaubte vielmehr, in ihm eine zugkräftige Symbolfigur für den politischen Kampf gefunden zu haben, die sich jedoch bald vom politischen Geschehen zurückziehen und den jüngeren Politikern das Feld überlassen würde ${ }^{\mathbf{7 5}}$. Diese Erwartung erwies sich als irrig. Der Arzt Banda wuchs bald weit über die ihm eigentlich zugedachte repräsentative Funktion hinaus und entwickelte sich im Laufe der Zeit immer stärker zu einem machiavellistischen Politiker mit großem Machtinstinkt und taktischem Geschick. Die Übernahme der Parteiführung hatte er zur Vorbedingung seiner Rückkehr nach Nyasaland gemacht. Als Parteiführer baute er den NAC - später die Malawi Congress Party (MCP) - zu einem schlagkräftigen Machtinstrument aus, das von keiner anderen Instanz im Lande mehr kontrolliert und reglementiert werden konnte: "The Party is supreme and no one is above the Party."76 Innerhalb der Partei stützte er sich vor allem auf die Frauen- und Jugendvereinigungen, die ihm seine besondere Aufmerksamkeit mit uneingeschränkter Loyalität dankten. Nachdem Banda 1961 zum Parteichef auf Lebenszeit ernannt wurde, fielen ihm später fast zwangsläufig auch die Ämter des Premierministers (1963) und des Präsidenten der Republik Malawi (1966) zu - eine Funktion, die ihm 1971 ebenfalls auf Lebenszeit zugesprochen wurde. In den letzten Jahren konzentrierte er immer mehr

\footnotetext{
73 "What was needed was a man of about 50 or 60 , who would command the respect of the moderates and yet be sympathetic to the aspirations of the younger men. The only possible candidatewas Dr. Banda . . ", Short, Philip, Banda, London and Boston, 1974, S. 83. 74 Zitiert nach: Short, Philip, Banda, a. a. O., S. 57.

75 Vgl. Short, Philip, Banda, a. a. O., S. 82/83.

76 Zitiert nach: Short, Philip, Banda, a. a. O., S. 169.
} 
Staatsämter auf seine eigene Person. Nach der Regierungsumbildung 1978 bekleidete er nicht nur die Ämter des Präsidenten, des Regierungs- und Parteichefs, sondern verwaltete zugleich die Ressorts des Äußeren, der Justiz, der Landwirtschaft und Bodenschätze sowie der Offentlichen Arbeiten. Angesichts solcher Machtfülle wird Malawi in der Literatur nicht nur als ,,one-party state“, sondern sogar als ,,one-man state“ bezeichnet ${ }^{77}$.

Die Zielstrebigkeit, die Banda bei der Erringung und Sicherstellung seiner eigenen Machtposition an den Tag legte, bewies er auch bei der Festlegung des politisch-ideologischen Kurses. Anfangs, während seiner Studien- und Berufszeit in Amerika und England, gehörte Banda zu den Gründungsmitgliedern des Panafrikanismus. Er stand damals in enger Verbindung mit führenden Vertretern der afrikanischen Unabhängigkeitsbewegung, wie Dr. Xuma, Julius Nyerere, Kwame Nkrumah u. a. Nach eigenen Aussagen war er in dieser Zeit ,the extremest of the extremists“.

In den ersten Jahren nach seiner Rückkehr änderte sich diese Haltung wenig. Mit äußerster Schärfe kämpfte er gegen die Föderation und für die Unabhängig Nyasalands und nahm dabei bewußt das Risiko einer Inhaftierung durch die britische Kolonialmacht auf sich: "They can do what they like to me. They can send me to prison. They can kill me. I will never give up my fight for freedom. We have to be prepared to go to prison. We must fill the prisons, millions of us. "78 Doch je mehr Entscheidungsgewalt Banda auf sich zog und je näher der Tag der Unabhängigkeit heranrückte, um so differenzierter und moderater wurden seine Ansichten und Aussagen: "I want you all to be peacefull . . . Do not trouble the Europeans. Do not trouble the Asians. Do not trouble the police . . . Do not spoil my work . . . Leave the trouble making to me . . . But I will not do it by throwing stones. I will do it with my tongue and brains."79 Mit Ubernahme der Regierungsgeschäfte wurde deutlich, daß er den von den meisten anderen Entwicklungsländern gleichsam vorgezeichneten Weg eines radikalen Antikolonialismus und Sozialismus nicht zu folgen bereit war. Vielmehr forderte er offen zur Imitation der Kolonisatoren auf. ,,Solange ich lebe, heißt die Losung für Malawi: Von den Weißen lernen. "80 Die entscheidende Weichenstellung erfolgte anläßlich einer schweren Kabinettskrise 1964, als er die von der Mehrheit seiner Ministerkollegen vorgeschlagenen Nationalisierungsprogramme ablehnte, nicht zu einer vorschnellen Afrikanisierung bereit war und ein sehr lukratives, allerdings an politische Bedingungen geknüpftes Kreditangebot der Volksrepublik China mit dem Hinweis der politischen Erpressung ausschlug. Hätten sich damals seine Widersacher im Kabinett mit einer stärker antikolonialistischen, antiwestlichen Orientierung durchgesetzt, wäre Malawi wahrscheinlich einen anderen Entwicklungsweg gegangen.

Der damals eingeschlagene pragmatisch-konservative Kurs seiner Politik ist vor dem Hintergrund seiner puritanischen Erziehung und seiner langjährigen Auslandserfahrung zu sehen. Die Philosophie Bandas ist geprägt durch prononcierten Individualismus, der auf Disziplin und Leistungswilligkeit des einzelnen abhebt: "So far as I know, in Africa, if they have any economic system at all, it is not capitalism, it is not communism, it is not socialism, it is definitely not statism. It is individualism . . . Independence does not mean that money will rain on our heads like manna from heaven. It means hard work in the fields with axes and hoes. The land is our gold-mine - and from it we must win our future."81

77 Ashford, Nicholas, The Adoration . .., a. a. O., und Short, Philip, Banda, a. a. O., S. 278.

78 Zitiert nach: Short, Philip, Banda, a. a. O., S. 104.

79 Malawi News: Great message of peace, 9. April 1960.

80 Zitiert nach: Knopp, Guido, Magier in Malawi, a. a. O.

81 Zitiert nach: Esterhuysen, Pieter, Malawi:The Doctor's Prescription, in: South African Journal of African Affairs, Vol. 9, No. 1, 1979, S. 24. 
Heute, nachdem der über 80jährige Präsident die Zügel des Landes fast zwei Jahrzehnte fest in der Hand hält, hat sich an der damals festgelegten ökonomisch-politischen Grundorientierung nichts geändert. Mit großem Geschick hat es Banda verstanden, in freiwilliger Abhängigkeit vom westlichen Ausland einen Kurs zu steuern, der dem Land ein Höchstmaß an Investitionsmitteln der internationalen Finanzgeber zufließen ließ ${ }^{\mathbf{8 2}}$.

Dieser lukrative Pragmatismus hat ihn zugleich zu einem außenpolitischen Balanceakt zwischen dem von Weißen dominierten Südafrika und den Staaten Schwarafrikas veranlaßt. Die Dialogpolitik mit Südafrika, die er allerdings mit großer Distanz betreibt, hat ihm und seinem Land große materielle Vorteile gebracht ${ }^{83}$. Die Republik Südafrika, die aus außen- und sicherheitspolitischen Motiven dringend an Stützpunkten in Schwarzafrika interessiert ist, war gern bereit, den Preis hoher Infrastrukturinvestitionen - z. B. den Bau der neuen Hauptstadt Lilongwe - zu zahlen, wenn das weiße Minderheitsregime dadurch nur einen kleinen Schritt in Richtung auf eine größere internationale Anerkennung vorankäme ${ }^{84} . \mathrm{Ob}$ die gewagte Rechnung Bandas allerdings auch in Zukunft aufgeht, wird sich noch zeigen müssen. Nach der Unabhängigkeit Zimbabwes unter der Führung Robert Mugabes versucht sich Malawi neuerdings wiederum stärker auf seine schwarzafrikanische Solidarität gegenüber der weißen Vorherrschaft im Süden Afrikas zu besinnen ${ }^{\mathbf{8 5}}$.

Die eigenwillige und im schwarzafrikanischen Kontext außergewöhnliche Politik Bandas blieb nicht unwidersprochen. Von Anfang an hatte er mit Andersdenkenden, vor allem mit politischen Rivalen zu kämpfen. Dabei entwickelte der alternde Präsident einen unerwartet großen Machtinstinkt. Spätestens während der Kabinettskrise von 1964 zeigte er, daß er beabsichtigte, nach eigenen Vorstellungen ganz allein die Geschicke des Landes zu lenken. Sechs Minister, darunter seine engsten Mitstreiter im Unabhängigkeitskampf, Chiume und Chipembere, wurden entlassen und mußten außer Landes gehen. In der Folgezeit verhärtete sich das politische Klima. Übertriebener Argwohn vor äußeren und inneren Bedrohungen führte zu immer unkontrollierteren Formen polizeistaatlicher Willkür. Tatsächliche oder nur vermeintliche Oppositionelle wurden mit Billigung des Präsidenten rücksichtslos verfolgt. Ethnische, rassische und religiöse Vorurteile spielten trotz anderslautender Erklärungen dabei ein Rolle. Verhaftet und mißhandelt wurden vor allem Angehörige derjenigen Stämme, denen der Präsident - er ist Chewa - nicht angehört ${ }^{86}$, oder die Zeugen Jehovas, die es aus religiösen Gründen ablehnten, sich der Zwangsmitgliedschaft der Einheitspartei MNC zu unterwerfen ${ }^{87}$. Bei der Verfolgung politischer Gegner stützte sich Banda besonders auf die Jungen Pioniere, die Jugendorganisation der Einheitspartei, die als persönliches Einsatzkommando des Präsidenten größere Kompetenzen erhalten hatten als die Polizei ${ }^{88}$. Bandas autoritärer Regierungsstil ist durch einen puritanischen Paternalismus geprägt: In seinen Reden verweist er immer wieder auf die Grundtugenden: unity - loyality - obedience - discipline. Auf Einhaltung solcher Werte pocht er in der Art eines absolutistischen Landesfürsten, wobei er sich selbst nicht unbedingt an die bestehenden Gesetze und Regelungen hält. Vielmehr beliebt er kraft eigener Autorität Verfügungen zu treffen, so daß demokrati-

82 Vgl. oben Kapitel 2.2 und Ubersicht 2.

83 Zur Außenpolitik Malawis und den Kontakten zu Südafrika vgl. insbesonders: Harding, Leonhard, Afrikanische Politik im Südlichen Afrika, München/Mainz, 1975, Kap. 5. Speck, Samuel W. Jr., Malawi and the Southern African Complex, in: Christin P. Potholm and Richard Dale (ed.), Southern Africa in Perspective, New York/London, 1972, S. 46-58. McMaster, Carolyn, Malawi - Foreign Policy and Development, London, 1974, Kap. 5-7.

84 Malawi ist das einzige schwarzafrikanische Land nördlich des Sambesi, das volle diplomatische Beziehungen mit Südafrika unterhält.

85 Banda hatte mit seiner Unterstützung Muzorewas zunächst aufs falsche Pferd gesetzt. Nun bemüht er sich durch stärkere Distanz zu Südafrika, diesen Fehler auszubügeln.

86 Vgl. McMaster, Carolyn, Malawi, a. a. O., S. 64 f.

87 Die Zahl der ohne Gerichtsurteil unbefristet Internierten lag Mitte der 70er Jahre bei ca. 2000 Personen.

88 Williams zitiert Banda: "The Young Pioneers cannot be arrested by any policeman without my consent." In: Williams, T. David, Malawi: The Politics of despair, Ithaka/London, 1978, S. $227 \mathrm{f}$. 
sche und rechtsstaatliche Institutionen zur Farce werden. Im Einparteienstaat Malawi kann heute von demokratischen Willensbildungs- und Entscheidungsprozessen nicht mehr die Rede sein ${ }^{89}$. "The Malawi system, the Malawi style is that Kamuzu says it's just that, and then it's finished. Whether anyone likes it or not, that is how it is going to be here. No nonsense, no nonsense. You can't have everybody deciding what to do."90

Es ist die Person Bandas, um die sich seit fast 20 Jahren in Malawi alles dreht. Konkurrenz ist nicht erlaubt - ein Nachfolger nicht in Sicht ${ }^{91}$.

\section{Der umstrittene Erfolg der malawischen Entwicklung}

Die Urteile über die malawische Entwicklung fallen sehr unterschiedlich aus. Von den einen wird das Land aufgrund seiner Abhängigkeit vom kapitalistischen Ausland und seiner pragmatischen Kooperation mit Südafrika scharf kritisiert und als Spielball der westlich-kolonialen Exploitationsinteressen betrachtet. Von den anderen wird die eigenwillige Entwicklungsstrategie Bandas hoch gelobt und der Wachstumserfolg des Landes als Ausnahmeerscheinung im Vergleich zu den Anstrengungen anderer Entwicklungsländer gewürdigt. Welche sozio-ökonomischen Fortschritte Malawi tatsächlich erzielt hat und inwiefern sie der breiten Masse der Bevölkerung zugute gekommen sind, ist im folgenden zu untersuchen.

\subsection{Zur gesamtwirtschaftlichen Entwicklung}

Trotz ungünstiger Ausgangsbedingungen ist es Malawi gelungen, überdurchschnittliche Wachstumsraten zu erzielen. Der von Banda bewußt forcierte Ausbau der materiellen Infrastruktur und die Betonung der ländlichen Entwicklungsprogramme unter dem Motto ,,food for the villages" haben die Gesamtentwicklung des Landes vergleichsweise schnell vorangetrieben $^{92}$. Das Bruttosozialprodukt ist seit 1964 jährlich im Durchschnitt um 4,6\% gestiegen. Basis für diesen Anstieg ist vor allem die landwirtschaftliche Produktion, die seit Mitte der 70er Jahre Zuwächse von mehr als $8 \%$ aufweist, so daß Malawi inzwischen nicht nur den Bedarf seiner Bevölkerung an Grundnahrungsmitteln decken kann, sondern darüber hinaus sogar Úberschüsse erwirtschaftet. Diese Ergebnisse rechtfertigen im nachhinein die entwicklungspolitische Grundentscheidung des Präsidenten. Allerdings ist bei einem weiteren Ausbau der cash-crop-Produktion im kleinbetrieblichen Bereich zu befürchten, daß es in Zukunft zu einem Produktionsrückgang bei Grundnahrungsmitteln kommt ${ }^{93}$, eine Tendenz, die durch die forcierte Förderung der Großplantagenwirtschaft in den letzten Jahren noch verstärkt wurde. Sie macht das Land nicht nur zunehmend von der Weltmarktentwicklung abhängig, sondern könnte auch den Selbstversorgungsgrad bei Grundnahrungsmitteln

\footnotetext{
89 Als Beispiel sei die „Parlamentswahl“ 1971 angeführt: Während einer Rede im Stadion von Blantyre ließ Banda seine Zuhörerschaft mit , Ja“- und ,Nein“-Rufen über eine von ihm vorgeschlagene Kandidatenliste abstimmen. Vgl. Elections: Malawian Style, Daily News, Dar es Salaam, 11. 6. 1978.

90 Zitiert nach: Short, Ph., Banda, a. a. O., S. 203

91 Jeder potentielle Nachfolger wird von Banda als Bedrohung angesehen. Eine Profilierung anderer Führungsfiguren ist nicht möglich. Die Folge ist, daß der Präsident seit Jahren nur von subalternen Ja-Sagern umgeben ist und über die tatsächliche Entwicklung im Lande gar nicht mehr wahrheitsgetreu informiert wird. Vgl. Malawi: Reverberations, in: Africa Confidential, Vol. 21, No. 7, 26. 3. 1980.

92 Im Entwicklungsprogramm 1978-1981 sind die Verkehrsausgaben mit 37,5 \% und die Mittel zur Agrarförderung mit 21 \% von insgesamt 417,6 Mio. MK angesetzt. Vgl. Development Division, March 1978.

93 Statistisches Bundesamt, Malawi, 1979, a. a. O., S. 7 f.
} 
verringern und den sozio-ökonomischen Dualismus zwischen kleinbäuerlicher Subsistenzwirtschaft und der großbetrieblichen Weltmarktproduktion verschärfen.

Eine größere Landflucht konnte bislang vermieden werden. Exakte Zahlen liegen allerdings nicht vor, da Wander- und Saisonarbeit sowie die versteckte Arbeitslosigkeit in der Subsistenzwirtschaft genaue Angaben erschweren. Man schätzt, daß 1978 etwa 340000 Beschäftigte in einem ständigen Arbeitsverhältnis (gegenüber 160000 in 1970) standen ${ }^{94}$. Diese Beschäftigten machen allerdings lediglich $10 \%$ der Bevölkerung aus. Die große Masse der Erwerbstätigen ist völlig oder größtenteils auf Einkommen aus der Subsistenzwirtschaft angewiesen $^{95}$.

Die Preisentwicklung erscheint bedrohlich, ist jedoch im internationalen Vergleich noch nicht dramatisch zu nennen. Für die letzten Jahre liegen drei Indizes vor: Der Lebenskostenindex für niedrige Einkommensbezieher betrug 1978185 (Basis $1970=100$ ), während der Lebenskostenindex für hohe Einkommensbezieher sich für den gleichen Zeitraum auf 231 belief ${ }^{96}$.

Diese Unterschiede sind darauf zurückzuführen, daß höherwertige Konsum- und Luxusgüter, die in der Regel importiert werden, einen überdurchschnittlichen Preisanstieg zu verzeichnen hatten. Das Preisniveau des Bruttosozialproduktes schließlich ist seit 1973 durchschnittlich um $12 \%^{97}$ jährlich gestiegen, ein Anstieg, der insbesondere auf die gestiegenen Erdölpreise zurückzuführen ist.

Zusammenfassend ist festzustellen, daß die rurale Entwicklungsstrategie Malawis den sozio-ökonomischen Bedingungen des Landes in der Vergangenheit weitgehend gerecht geworden ist. Malawi ist eines der wenigen Entwicklungsländer, welches trotz einer jährlichen Zuwachsrate der Bevölkerung von 2,9\% ${ }^{98}$ den Eigenbedarf an Grundnahrungsmitteln deckt und - gemessen an den gesamtwirtschaftlichen Indikatoren Wachstumsrate des Bruttosozialprodukts, Beschäftigungsstand sowie Preisniveauentwicklung - eine überdurchschnittliche Entwicklungsleistung aufzuweisen hat.

\subsection{Wirtschaftliche Entwicklung und Grundbedürfnisse}

Die erfolgreiche Maximierung der agrarischen Produktion Malawis kann als eine notwendige, aber nicht hinreichende Bedingung für die Befriedigung von Grundbedürfnissen der Bevölkerungsmehrheit betrachtet werden. Andere Aspekte der Grundbedürfnisbefriedigung müssen noch hinzugezogen werden.

Definition und exakte Analyse von Grundbedürfnissen und die Probleme ihrer Deckung werfen eine Reihe methodologischer und statistischer Probleme auf. Als bisher brauchbarstes Instrumentarium hat sich ein mehrdimensionaler Grundbedürfnis-Indikator, der vom amerikanischen Overseas Development Council entwickelte Physical Quality of Life Index

\footnotetext{
94 Etwa die Hälfte von ihnen arbeitete in der Landwirtschaft, dem Forst- und Fischereiwesen. Im produzierenden Gewerbe waren 69000 , im Handel, Banken- und Versicherungswesen etwa 35000 Erwerbstätige beschäftigt. Statistisches Bundesamt, Malawi, 1979 , a. a. O., S. 14.

95 Bundesstelle für Außenhandelsinformation, Malawi, 1977, a. a. O., S. 2. Das Statistische Bundesamt dagegen gibt die Zahl der bei den Arbeitsämtern registrierten Arbeitsuchenden mit 2280 (1976) an, vgl. Statistisches Bundesamt, Malawi, 1979, a. a. O., S. 14.

96 Ebenda, S. 21.

97 Ebenda, S. 23.

98 Republic of Malawi, Jenda Mzuzu Road, a. a. U., S. 27.
} 
$(\mathrm{PQLI})^{99}$ erwiesen. Er setzt sich aus den drei (Unter-)Indikatoren Säuglingssterblichkeit, Lebenserwartung und Alphabetenrate zusammen ${ }^{100}$.

$\mathrm{Da}$ absolute Werte zur Befriedigung physischer Grundbedürfnisse wenig aussagekräftig sind, wird im folgenden versucht, den PQL-Index für Malawi mit der Situation in ausgewählten schwarzafrikanischen Ländern in Beziehung zu setzen. Dabei ist es von Interesse, die gesellschaftspolitisch zum Teil anders orientierten Nachbarländer Moçambique, Tanzania und Sambia zu einem Vergleich heranzuziehen. Darüber hinaus schien es sinnvoll, mit Malawi - gemessen an der Bevölkerungszahl - vergleichbare Staaten wie Niger und Guinea in die Untersuchung einzubeziehen ${ }^{\mathbf{1 0 1}}$.

Es ist festzustellen, daß in der Regel zwischen der Höhe des Bruttosozialproduktes pro Kopf und der Befriedigung physischer Grundbedürfnisse eine positive Korrelation besteht. Länder mit niedrigem BSP pro Kopf wie Niger und Guinea haben niedrige PQL-Werte, während Sambia als Land mit dem höchsten BSP pro Kopf auch mit Abstand den höchsten PQL-Index aufweist (vgl. Übersicht 3). Die Republik Malawi ist jedoch ein Ausnahmefall, denn das Land hat zwar das niedrigste BSP pro Kopf der untersuchten Länder, nicht jedoch den niedrigsten PQL-Wert.

Dieses überraschende Ergebnis wird durch einen Vergleich der Ränge der ärmsten Länder der Welt, gemessen am BSP pro Kopf, und den Ländern mit den niedrigsten PQL-Werten bestätigt. Danach steht Malawi unter den ärmsten Ländern der Welt an 13. Stelle, d. h. ist das 13. ärmste Land der Welt, nimmt aber lediglich den 32. Rang bei den niedrigsten PQL-Werten ein.

99 Vgl. Sewell, John W. et al., The United States and World Development. Agenda 1977, New York/London, 1977, S. 147 f. Zur Berechnungsmethode vgl. Morris, David M., Measuring the Condition of the World's Poor. The Physical()uality of Life Index, New York/London, 1979, S. 41 f. Zur Messung der Veränderungsrate des PQLI vgl. Grant, James P., Disparity Reduction Rates in Social Indicators. A Prosposal for Measuring and Targeting Progress in Meeting Basic Needs, Overseas Development Council, Washington D. C., 1978.

100 Der PQL-Index hatsich gegenüber den herkömmlichen Kennziffern, insbesondere gegenüber der Messung des Bruttosozialproduktes pro Kopf, als vorteilhaft erwiesen. Die ihm zugrunde liegenden Daten sind in der Regel statistisch verfügbar und erlauben, da nicht ethnozentrisch, relativ objektive internationale Vergleiche (vgl. Morris, David M., a. a. ()., S. 22 f.). Im Gegensatz zu anderen Indikatoren mißt der PQL-Index nicht Input-Größen, sondern die Ergebnisse der Entwicklung in unterschiedlichen gesellschaftlichen Bereichen, so des Gesundheitswesens, des Bildungswesens, der Ernährungssituation, des Einkommensniveaus und des Zustandes der natürlichen Umwelt. Vgl. auch McLaughlin, Martin M, et al.. The United States and World Development. Agenda 1979, New York/London, 1979, S. $132 \mathrm{ff}$.

101 Es braucht nicht besonders betont zu werden, daß derartige Vergleiche wegen der unterschiedlichen historischen, geographisch-klimatischen und demographischen Voraussetzungen immer problematisch bleiben werden. 


\section{Ubersicht 3}

Wirtschaftliche Entwicklung und physische Grundbedürfnisse in ausgewählten

\begin{tabular}{|c|c|c|c|c|c|c|c|}
\hline Land & $\begin{array}{c}\text { Bevölkerung } \\
1978 \\
\text { (in Mill.) }\end{array}$ & $\begin{array}{c}\text { BSP } \\
\text { pro } \\
\text { Kopf } \\
\text { (in Dollar) }\end{array}$ & $\begin{array}{l}\text { PQL- } \\
\text { Index }\end{array}$ & $\begin{array}{c}\text { Rang }{ }^{103} \text { unter } \\
\text { Ländern mit } \\
\text { niedrigstem } \\
\text { BSP pro } \\
\text { Kopf }\end{array}$ & $\begin{array}{c}\text { Rang }^{102} \text { unter } \\
\text { Ländern mit } \\
\text { niedrigstem } \\
\text { PQL-Index }\end{array}$ & $\begin{array}{c}\text { Zuwachsrate } \\
\text { des BSP pro } \\
\text { Kopf } \\
1970-1975 \\
\text { (in \%) } \\
\end{array}$ & $\begin{array}{c}\text { Lebens- } \\
\text { erwartung } \\
\text { bei Geburt } \\
\text { (in Jahren }^{10}\end{array}$ \\
\hline Malawi & 5.4 & 140 & 30 & 13. & 32. & 7.0 & 43 \\
\hline Mocambique & 9.9 & 170 & 27 & 24. & 22. & -2.6 & 44 \\
\hline Sambia ${ }^{107}$ & 5.5 & 440 & 44 & 54. & 44. & 0.9 & 44 \\
\hline Tanzania & 16.5 & 180 & 30 & 31. & 33. & 2.9 & 44 \\
\hline Niger & 5.0 & 160 & 14 & 18. & 2. & -2.8 & 39 \\
\hline Guinea & 4.8 & 150 & 20 & 16. & 12. & 1.3 & 41 \\
\hline $\begin{array}{l}\text { Afrikanische } \\
\text { Least Deve- } \\
\text { loped Coun- }{ }^{108} \\
\text { tries insges. }\end{array}$ & 241.8 & 191 & 31 & & & 0.8 & 45 \\
\hline
\end{tabular}

102 Zusammengestellt nach: Mc Laughlin, Martin M. et al. The United States and World Development. Agenda 1979, New York 1979, p. 156 f (außer E 103 Berechnet nach: Morris, David Morris Measuring the Condition of the World's Poor. The Physical Quality of Life Index, New York 1979 p. $138 \mathrm{f}$ (Die und dem PQL-Index dieser Länder von 1976 festgelegt). Den 1. Rang nimmt das Land mit niedrigstem BSP pro Kopf bzw. niedrigstem PQL-Index 104 Jeweils letzte verfügbare $\mathrm{Zahl}$

105 Anteil der Erwachsenen über 15 Jahre mit Schreib- und Lesekenntnissen. Jeweils letzte verfügbare Zahl

106 Weltbank (Hrsg.) Weltentwicklungsbericht 1979, Washington D. C. 1979, S. 186

107 Zählt zu den ,Lower-Middle Income Countries' mit einem BSP pro Kopf zwischen 300 und 699 Dollar 108 BSP pro Kopf unter 300 Dollar 
Die Republik Malawi ist damit das einzige unter den betrachteten Ländern, das bei der Befriedigung physischer Grundbedürfnisse einen besseren Rang einnimmt als beim Bruttosozialprodukt pro Kopf. Die Ursache dieser Entwicklung dürfte primär in der relativ günstigen Ernährungssituation der malawischen Bevölkerung zu suchen sein. So zeigt eine vergleichende Analyse, daß in Malawi das tägliche Kalorienangebot den Bedarf übersteigt. Damit ist es Malawi neben Madagaskar als einzigem schwarzafrikanischen Land gelungen, die Grundernährung seiner Bevölkerung und damit wichtige Teile des physischen Grundbedarfs zu decken ${ }^{109}$.

\section{Zusammenfassung}

Die Republik Malawi nimmt im schwarzafrikanischen Kontext eine Sonderstellung ein. Der patriarchalischen Staatsführung unter Banda ist es gelungen, durch eine Kombination freiwilliger Dependenz nach außen und autoritäre Grundbedürfnisstrategien nach innen die physischen Lebensbedingungen der breiten Bevölkerungsmehrheit zu verbessern. Die bestehende Abhängigkeit Malawis ist von seinem Präsidenten bewußt gewollt und durch ihn entscheidend geprägt worden. Wenn auch nicht unbedingt ,,Männer Geschichte machen“ so ist Geschichte auch nicht ,ohne Männer" denkbar. Wie die Statistiken zeigen, hat von dieser selbstgewählten Abhängigkeit nicht nur die Staatsoligarchie profitiert, sonderns es ist offenbar gelungen, auch die materiellen Bedürfnisse breiter Bevölkerungsschichten zu befriedigen. Damit ist allerdings keine Aussage über eine Befriedigung zentraler, immaterieller Bedürfnisse, wie etwa die Durchsetzung politischer Partizipation oder die Wahrung allgemeiner Menschenrechte, gemacht.

Autoritäre Basic-Needs-Strategien ,,from above“ scheinen, sofern dieses Ergebnis generalisiert werden kann, für die ärmsten Länder der Welt durchaus geeignet zu sein, die zentralen physischen Grundbedürfnisse ihrer Bevölkerung decken zu helfen. Ob es allerdings langfristig möglich sein wird, die physischen Grundbedürfnisse ohne ausreichende Berücksichtigung der psychischen zu befriedigen, scheint sehr zweifelhaft. Alle Erfahrungen deuten darauf hin, daß mit zunehmendem materiellen Wohlstand die Forderungen nach nicht-materiellen Gütern, d. h. vor allem nach Partizipation, ansteigen ${ }^{\mathbf{1 1 0}}$. Der Weg aus sozio-ökonomischer Unterentwicklung und Armut ist also erst zur Hälfte beschritten. Allerdings ist diese Etappe bereits als Erfolg zu bewerten. Denn schon Engels betonte in seiner Grabrede für Karl Marx: ,Wie Darwin das Gesetz der organischen Natur, so entdeckte Marx das Entwicklungsgesetz der menschlichen Geschichte: die bislang unter ideologischen Überwucherungen verdeckte einfache Tatsache, daß die Menschen vor allen Dingen zuerst essen, trinken, wohnen und sich kleiden müssen, ehe sie Politik, Wissenschaft, Kunst, Religion usw. betreiben können." 111

\footnotetext{
109 Der mögliche Einwand, die durchschnittliche Deckung des Kalorienbedarfs pro Tag sage noch nichts über die Struktur und über die Verteilung des Nahrungsmittelkonsums aus, trifft nicht; denn es wurden nicht das Kalorienangebot, sondern die PQL-Werte verglichen, in die distributive Gewichte und Konsumstruktur über die (Unter-)Indikatoren des PQL-Index Lebenserwartung und Säuglingssterblichkeit eingehen.

110 Vgl. dazu Lindner, D., Theorie der Revolution, München, 1972.

111 Engels, Friedrich, Das Begräbnis von Karl Marx, in: Der Sozialdemokrat, Nr. 13, 22. März 1883; abgedruckt in: Marx, Karl, Engels, Friedrich, Ausgewählte Schriften in zwei Bänden, Band II, 18. Aufl., Berlin, 1970, S. 152.
} 
both at law and in general political character, is then discussed in comparative perspective. This is followed by a fairly detailed treatment, again in comparative perspective, of the nature and scope of the executive power. This aspect is considered, first, under the head of prerogative and other superior discretionary competence; secondly, with regard to the subordinate administrative agencies of the Executive; and lastly, in terms of the extrajuridical competence (or capacity?) which the executive organ may come to enjoy, not so much in virtue of legal prescriptions, but, rather, purely due to the historical circumstances wherein the structure and process of leadership have been shaped and in which, in particular, accession to power has taken place.

It would emerge from the study that, since independence, in anglophonic as in francophonic Africa, a fundamental transformation of function, vis-à-vis the western-derived constitutional concepts, has come about. While the basic constitutional terminology remains the same, the constitutional structure has been readapted to the dictates of a new power-situation - a situation largely owing to common problems of nation building and economic development. In this new power structure the Executive is the all dominant organ, a fact which at once poses the most difficult questions in relation to any attempts at power control; the western-derived control procedures, although still forming part of the constitutional landscape, were obviously not designed with the apparent trends in view!

\section{The Development of the Republic of Malawi: A Strategy between Voluntary Depend- ency and Authoritarian Satisfaction of Basic Needs \\ By Gerald Braun and Heribert Weiland}

The development of the Rupublic of Malawi is judged variously: While the UN classifies Malawi as a least developed country, other development experts see this mini-state as a prosperous police state or as "la Suisse d'Afrique".

The article attempts to show empirically the external dependency of Malawi by using different dependency concepts, such as external sensitivity, unequal exchange, external determination, unequal cost-benefit distribution.

The authors illustrate that all forms of dependency can be demonstrated empirically. They note that Malawi's colonial dependence on Great Britain has been substituted by a new dependence on the Republic of South Africa.

This dependence, however, is very much different from the excolonial relationships of other African states, because it has caused a transfer of resources in favour of Malawi. This paradoxical development is due to a strategy of voluntary dependency, which has been pursued by the authoritarian leader of Malawi, President Dr. Kamuzu H. Banda. It is this aspect of voluntary dependency, which is not sufficiently seen and explained by the economically biased dependency concepts.

Although this voluntary dependency proved to be very profitable for the state bureaucracy in Malawi, it also did not harm the mass of the population. On the contrary, according to the Physical Quality of Life Index, Banda's development policy has led to a degree of satisfaction of physical basic needs for the masses in Malawi which ranks significantly above the level of comparable African states. If one tries to generalize these findings, it seems that authoritarian basic needs strategies can lead to a satisfaction of the physical needs of the population. However, it is doubtful whether the authoritarian satisfaction of physical needs can solve the problem of underdevelopment in the long run. There is empirical evidence that, with rising standards of living, the demands for non-material goods - especially for participation - increase as well. 